\title{
ON SEPARATING TRANSCENDENCY BASES FOR DIFFERENTIAL FIELDS
}

\section{A. SEIDENBERG}

Let $F$ be an arbitrary ordinary differential field ${ }^{1}$ of characteristic $p \neq 0$, and let $F\left\langle u_{1}, \cdots, u_{n}\right\rangle$ be a differential extension field of $F$ of degree of differential transcendency $t$. In $[1$, p. 189], we stated a theorem which, as far as wording is concerned, is analogous to a wellknown theorem of S. MacLane in ordinary algebra. This theorem of ours states that if $F\left\langle u_{1}, \cdots, u_{n}\right\rangle / F$ is separable, then some $t$ of the $u_{i}$ form a separating transcendency basis, i.e., for an appropriate relettering of the $u_{i}, F\left\langle u_{1}, \cdots, u_{n}\right\rangle$ is separable over $F\left\langle u_{1}, \cdots, u_{t}\right\rangle$. The object of the present note is to establish the following stronger version of that theorem. ${ }^{2}$

Theorem. If $F\left\langle u_{1}, \cdots, u_{n}\right\rangle / F$ is separable, then any transcendency basis of $F\left\langle u_{1}, \cdots, u_{n}\right\rangle / F$ is also a separating transcendency basis.

Proof. We first prove that any $t$ of the $u_{i}$ which form a transcendency basis also form a separating transcendency basis. The theorem will then follow for any transcendency basis $v_{1}, \cdots, v_{t}$ since obviously we may include the $v_{j}$ amongst the $u_{i}$.

For $t=0$, there is nothing to prove. Confining ourselves to transcendency bases selected from the $u_{i}$, the theorem is also immediate for $t=n$. Consider next the case $t=n-1$, and let $u_{1}, \cdots, u_{n-1}$ be algebraically independent over $F$. By $[1$, p. 188, Theorem 6, Corollary], the $u_{i j}, i=1, \cdots, n-1 ; j=0,1, \cdots$, are algebraically independent over $F$. By the definition in $\left[1\right.$, p. 183], $F\left\langle u_{1}, \cdots, u_{n}\right\rangle$ is finite over $F\left\langle u_{1}, \cdots, u_{n-1}\right\rangle$, so for some $d, u_{n d}$ is algebraic over $F\left\langle u_{1}, \cdots, u_{n-1}\right\rangle\left(u_{n 0}, \cdots, u_{n, d-1}\right)$. Let $d$ be minimal, i.e., $u_{i j}, u_{n k}$, $i=1, \cdots, n-1 ; j=0,1, \cdots ; k=0, \cdots, d-1$, are algebraically

Presented to the Society, December 29, 1954; received by the editors October 27, 1954.

${ }^{1}$ Definitions, notation, and terminology will be as in [1].

2 The proof of the weaker theorem in $[1 ;$ p. 189], though essentially correct, is too compressed; and we would like to add one remark to that proof. Let $G, U_{n r}$ be as in the proof; replacing $G$ by a derivative if necessary, we may suppose $G$ involves no proper derivative of $U_{n r}$. As $G\left(u_{1}, \cdots, u_{n-1} ; u_{n 0}, \cdots, u_{n, r-1}, U_{n r}\right)=0$ is not necessarily a defining equation for $u_{n r}$, the separability of $u_{n r}$ over $F\left\langle u_{1}, \cdots\right.$, $\left.u_{n-1}\right\rangle\left(u_{n 0}, \cdots, u_{n, r-1}\right)$ does not yet follow from the form of $G$. That separability would follow, however, if we had that $\partial G / \partial U_{n r} \neq 0$ for $U=u$ : this we have because of the minimal degree of $G$. With this additional point in mind, it is not difficult to fill the slight gaps which occur in the proof as it now stands in [1]. 
independent over $F$, but $u_{n d}$ is algebraically dependent on this set over $F$. Let $A$ be the set of polynomials $\{G\}$ in the polynomial ring $F\left\{U_{1}, \cdots, U_{n}\right\}$ such that $G \neq 0, G$ is of degree 0 in $U_{n i}, i>d$, and $G\left(u_{1}, \cdots, u_{n}\right)=0$. Let $B$ be the subset of $A$ consisting of the polynomials of minimum total degree. Let $G \in B$. The separability of $F\left\langle u_{1}, \cdots, u_{n}\right\rangle / F$ implies that $G \notin F\left[\cdots, U_{i j}^{p}, \cdots\right]$. Not all the $U_{n i}, i \leqq d$, occurring in $G$ occur with exponent divisible by $p$. In fact, assume otherwise. Since not all the exponents occurring in $G$ are divisible by $p$, at least one of the $U_{j k}, j=1, \cdots, n-1$, say $U_{1 h}$, occurs in $G$ with exponent not divisible by $p$; we may suppose that derivatives of $U_{1 h}$, if they occur in $G$, occur with exponents divisible by $p$. The derivative $G^{\prime}$ of $G$ also is in $A$ and in $B$; so replacing $G$ by a derivative if necessary, we may suppose $G$ involves no proper derivative of $U_{1 h}$. With these assumptions on $G$, we have: (1) degree of $G^{\prime}$ in $U_{1, h+1}$ is 1 , degree of $G^{\prime}$ in $U_{1 j}, j>h+1$, is 0 ; (2) coefficient of $U_{1, h+1}$ in $G^{\prime}$ does not vanish at $U=u$, since it is of too small degree to be in $A$. Hence $u_{1 j} \in F\left\langle u_{2}, \cdots, u_{n-1}\right\rangle\left(u_{n 0}^{p}, \cdots, u_{n d}^{p} ; u_{10}, \cdots\right.$, $\left.u_{1, h}\right), j \geqq 0$. Since $F\left\langle u_{1}, \cdots, u_{n}\right\rangle / F\left\langle u_{1}, \cdots, u_{n-1}\right\rangle$ is finite, for some $r, r \geqq d$, we have $u_{n j} \in F\left\langle u_{1}, \cdots, u_{n-1}\right\rangle\left(u_{n 0}, \cdots, u_{n r}\right), j \geqq 0$. This last field may be written as $F\left\langle u_{2}, \cdots, u_{n-1}\right\rangle\left(u_{n 0}, \cdots, u_{n r} ; u_{10}, \cdots, u_{1 h}\right)$, whence $F\left\langle u_{1}, \cdots, u_{n}\right\rangle / F\left\langle u_{2}, \cdots, u_{n-1}\right\rangle$ is finite. This contradicts the assumption $t=n-1$. Hence for any given $G \in B$, at least one $U_{n j}$, $j \leqq d$, occurs with exponent not divisible by $p$. Differentiating $G$ sufficiently often we may suppose that $U_{n d}$ occurs in $G$ with exponent not divisible by $p$. Since $u_{i j}, u_{n k}, i=1, \cdots, n-1 ; j=0,1, \cdots$; $k=0, \cdots, d-1$, are algebraically independent over $F$, we have that $G\left(u_{i j}, u_{n k}, U_{n d}\right)=0$ is an irreducible (separable) equation for $u_{n d}$ over $F\left(u_{i j}, u_{n k}\right)$. Hence $F\left\langle u_{1}, \cdots, u_{n}\right\rangle$ is separable over $F\left\langle u_{1}, \cdots, u_{n-1}\right\rangle$. This completes the proof for $t=n-1$.

For $0<t<n-1$, we apply the Theorem of the Primitive Element. In the application, no separability condition is required (as in ordinary algebra-see the remarks in $[1$, p. 183 , bottom of page $]$ ), but we do need to know, or rather, it would be sufficient to know, that $F\left\langle u_{1}, \cdots, u_{t}\right\rangle$, where $u_{1}, \cdots, u_{t}$ is any given transcendency basis, has no finite linear basis over its field of constants. Even if $F\left\langle u_{1}, \cdots, u_{t}\right\rangle$ had a finite linear basis over its field of constants, we could overcome this difficulty by the well-known device of adjoining an appropriate nonconstant element to $F\left\langle u_{1}, \cdots, u_{n}\right\rangle$. Here we may as well determine the constants of $F\left\langle u_{1}, \cdots, u_{t}\right\rangle$. If $F_{0}$ is the constant-field of $F$, then we shall see that the constant field of $F\left\langle u_{1}, \cdots\right.$, $\left.u_{t}\right\rangle$ is $F_{0}\left(\cdots, u_{i j}^{p}, \cdots\right)$. Assuming this for a moment we see that $F\left\langle u_{1}, \cdots, u_{t}\right\rangle$ has no finite linear basis over its field of constants, 
whence $F\left\langle u_{1}, \cdots, u_{n}\right\rangle=F\left\langle u_{1}, \cdots, u_{t} ; w\right\rangle$. By the case $t=n-1$, then, the basis $u_{1}, \cdots, u_{t}$ is separating.

Since $F\left\langle u_{1}, \cdots, u_{t}\right\rangle / F$ is separable, the $u_{i j}$ are, as previously mentioned, algebraically independent: the converse is immediate.

Lemma. Let $F$ be a differential field of characteristic $p \neq 0, F_{0}$ its field of constants, and assume that $F\left\langle u_{1}, \cdots, u_{t}\right\rangle / F$ is separable and of degree of differential transcendency $t$. Then $F_{0}\left(\cdots, u_{i j}^{p}, \cdots\right)$, $i=1, \cdots, t ; j=0,1, \cdots$, is the field of constants of $F\left\langle u_{1}, \cdots, u_{t}\right\rangle$.

Proof. Let $P(u) / Q(u) \in F\left\langle u_{1}, \cdots, u_{t}\right\rangle$ be a constant $\neq 0$, where $P(u), Q(u)$ are elements of the polynomial ring $F\left\{u_{1}, \cdots, u_{t}\right\}$, and $P$ and $Q$ have no common factor of positive degree. We first assert that $P, Q \in F\left[\cdots, u_{i j}^{p}, \cdots\right]$. For suppose this is not the case, and say $Q \notin F\left[\cdots, u_{i j}^{p}, \cdots\right]$. Then $Q^{\prime}$ is not zero, and $P / Q=P^{\prime} / Q^{\prime}$. Since degree of $P=$ degree of $P^{\prime}$ and degree of $Q=$ degree of $Q^{\prime}$, we get $P^{\prime}=d P, Q^{\prime}=d Q$ for some $d \in F, d \neq 0$. Repeating the argument, we get $P^{(i)}=d_{i} P, Q^{(i)}=d_{i} Q$, where $d_{i} \in F$ and the superscript indicates the $i$ th derivative. Since $Q^{(i)}$ for sufficiently high $i$ involves some $u_{j k}$ not occurring in $Q$, we have a contradiction. Thus $Q \in F[\cdots$, $\left.u_{i j}^{p}, \cdots\right]$, and similarly for $P$. Let $P=\sum a_{i} \pi_{i}^{p}, Q=\sum b_{i} \pi_{i}^{p}$, where $a_{i}, b_{i} \in F, a_{i} b_{i} \neq 0$, and the $\pi_{i}$ are power products of the $u_{j k}$ with $\pi_{i} \neq \pi_{j}$ for $i \neq j$. If $Q^{\prime}=0$, then each $b_{i}$ is a constant, since $Q^{\prime}=\sum b_{i}^{\prime} \pi_{i}^{p}$ $=0$; and likewise the $a_{i}$ are constant; so $P(u) / Q(u)$ has the required form if $Q^{\prime}=0$. Assume $Q^{\prime} \neq 0$ : then as above we have $P^{\prime}=d P, Q^{\prime}=d Q$, $d \in F, d \neq 0$. This yields $a_{i}^{\prime}=d a_{i}$, whence any two $a_{i}$ have a constant ratio. Thus $P=e \sum a_{i} \pi_{i}^{p}, Q=f \sum b_{i} \pi_{i}^{p}$, where now the $a_{i}, b_{i}$ are in $F_{0}$. Since $P / Q$ and $\sum a_{i} \pi_{i}^{p} / \sum b_{i} \pi_{i}^{p}$ are constants, so is $e / f$. Thus $P / Q$ has the desired form. This completes the proof.

\section{REFERENCE}

1. A. Seidenberg, Some basic theorems in differential algebra (characteristic $p$, arbitrary), Trans. Amer. Math. Soc. vol. 73 (1952) pp. 174-190.

University of California, Berkeley 